\title{
Involvement of the red light receptors phytochrome A and phytochrome $B$ in the regulation of gene expression of the plastid transcription apparatus by cytokinin during de-etiolation of $A$. thaliana
}

\author{
Doroshenko A.S. ${ }^{1 *}$, Malyukova A.M. ${ }^{1,2}$, Danilova M.N. ${ }^{1}$ \\ ${ }^{1}$ Timiryazev Institute of Plant Physiology, RAS, Moscow, Russia \\ ${ }^{2}$ Moscow State University, Moscow, Russia \\ * email: anastasiya04101993@gmail.com
}

The development of chloroplast from etioplast during de-etiolation is based on a lightdependent change in the expression of the plastid and nuclear genomes. Activation of plastid gene transcription is caused by the complication of the plastid transcription apparatus (PTA). In etioplasts, the monosubunit RNA-polymerase NEP, which is represented by two enzymes RPOTp and RPOTmp, exhibits a greater transcriptional activity, while the multisubunit RNA-polymerase PEP, represented by the core subunits and sigma-factor, has a lower transcriptional activity. Under white light PEP is activated due to association with PAP proteins. In addition to light, phytohormones also affect the architecture of PTA during the de-etiolation. In particular, cytokinins stimulate the accumulation of transcripts of some PTA genes under white light, but the molecular mechanism is not completely known. We suggested that the positive effect of cytokinin on the expression of PTA genes may be mediated by the red light receptors phytochromes. Using PCR after reverse transcription, we investigated the regulation of the expression of PTA genes by red light and cytokinin in wild type Landsberg erecta (Ler) and mutant phyAphyB seedlings. Compared to the white light, red-light caused a smaller increase in the mRNA level of some PTA genes in Ler. Cytokinin stimulated the growth of mRNA level of some PTA genes under both white and red light, however under red light a weaker response was observed. In contrast to Ler, white and red-light exposure of phyAphyB resulted in a smaller rise of transcripts level of some PTA genes. Cytokinin treatment of phyAphyB under both red and white light stimulated the accumulation of transcripts of some PTA genes, however their level was lower than in Ler. These results indicate the involvement of phytochrome A and B in cytokinindependent de-etiolation of A.thaliana.

Acknowledgments: This research is supported by the RFBR (grants No. 19-34-90183 and No. 20-04-00294A). 